\title{
Spermatogenesis in retinol-deficient rats maintained on retinoic acid
}

\author{
M. E. A. B. van Beek* and M. L. Meistrich $\dagger$ \\ Department of Experimental Radiotherapy, The University of Texas M. D. Anderson Cancer \\ Center, 1515 Holcombe Boulevard, Houston, Texas 77030, USA
}

\begin{abstract}
Summary. Rats maintained on a diet deficient in retinol and retinoic acid were given a diet containing retinoic acid for 21-29 days after the start of weight loss. The testes of four of these rats were studied. Spermatogonia of all types were observed, though in lower numbers than in controls, and their mitotic activity was normal. Normal preleptotene spermatocytes were encountered, but no normal spermatocytes in further stages of development were seen. Pale cells that appeared to be in prophase were observed. It was concluded that, in retinol-deficient rats maintained on retinoic acid, the spermatogonial population is qualitatively normal, but quantitatively subnormal, while spermatocyte development is qualitatively and quantitatively abnormal. No evidence of spermatogonial arrest or any other form of synchronization was found in testes of these rats, but when the remaining rats were injected with retinol, the seminiferous epithelium did show stage synchronization at 36 and 128 days after the injection.
\end{abstract}

Keywords: spermatogenesis; retinol; rat

\section{Introduction}

Retinol (vitamin A) is necessary for normal growth and health of rats (Coward et al., 1969). The systemic symptoms of retinol deficiency may be relieved by supplementing the diet with retinoic acid (retinol-deficient, retinoic-acid-maintained, or RD-RAM) (Howell et al., 1963), but the testes remain underdeveloped. The regression of the seminiferous epithelium can be reversed by resupplementation with retinol (Huang \& Hembree, 1979).

The seminiferous epithelium of rats has a cycle consisting of 14 cellular associations or stages, which in control rats occur in a frequency related to the duration of each stage (Leblond $\&$ Clermont, 1952a). Recently, it has proved possible to synchronize the epithelium in only a few sequential stages. Several groups have reported that such stage synchronization occurs in testes of rats that, after becoming retinol deficient, either without (Morales \& Griswold, 1987; Morales et al., 1989; Bartlett et al., 1989, 1990) or with (Huang et al., 1990; van Beek \& Meistrich, 1991) retinoic acid maintenance, were resupplemented with retinol.

Van Pelt \& de Rooij (1990) and Ismael et al. (1990) have investigated the spermatogonial population in totally retinoid-deficient animals. Both studies found that the undifferentiated $A$ spermatogonia were arrested and did not form differentiating $\mathrm{A}_{1}$ spermatogonia, and concluded that the injection of retinol relieved this arrest, causing all A spermatogonia to start the series of mitoses as $A_{1}$ spermatogonia, resulting in stage synchronization of the testis. However, other studies have indicated the presence of differentiating spermatogonia in the seminiferous epithelium

*Present address: Department of Environmental Toxicology, The University of California at Davis, Davis, CA 95616, USA.

†Reprint requests. 
of rats that, after becoming retinol-deficient, had been maintained on a retinoic acid-containing diet (Huang \& Hembree, 1979; Unni et al., 1983; Huang et al., 1990), and that stage synchrony can also be achieved in this situation (Huang et al., 1990; van Beek \& Meistrich, 1991). Thus, it is possible that the arrest of the A spermatogonia is incidental and not critical for stage synchrony to be established, and that stage synchrony is induced by another, as yet unknown, mechanism.

In this study we further examined the proliferation of spermatogonia in RD-RAM rats using testicular cross-sections and tubular whole mounts. The distribution of different types of differentiating spermatogonia which are normally found in different stages was also examined, and compared with the high degree of stage synchronization subsequently obtained after injection of retinol. On basis of these results, an alternative hypothesis for the mechanism of retinol-induced stage synchronization of the testis will be presented.

\section{Materials and Methods}

\section{Diets}

Two custom AIN-76 diets without retinol were obtained from ICN (Cleveland, Ohio). Diet RD was deficient in retinol and retinoic acid; diet RA 10 was identical, but $10 \mathrm{mg}$ retinoic acid per $\mathrm{kg}$ of food was added.

\section{Experimental animals}

Rats were obtained at 20 days of age from Harlan Sprague-Dawley (Indianapolis, IN). Upon arrival in our laboratory, the rats were fed diet RD, to exhaust their reserves of retinol. This induces retinol-deficiency, which causes regression of the seminiferous epithelium as well as systemic effects. At 55-60 days of age, the rats became critically deficient in retinol as judged from their appearance (listless, huffed fur) and weight loss. At 69 or 77 days of age, at which time the seminiferous epithelium should be regressed (Mitranond et al., 1979), they were placed on diet RA10 for 21 or 29 days, until 98 days of age.

At 98 days of age, four rats were randomly selected from this group of nine rats and killed to study the RD-RAM seminiferous epithelium. The five remaining rats were injected with retinol and subsequently fed a diet enriched in retinol, according to the protocol of Morales \& Griswold (1987). Rats I and 3 of the present study had received diet RA 10 for 21 days (RA-maintenance initiated at Day 77), while rats 2 and 4 had received diet RA 10 for 29 days (RA-maintenance initiated at Day 69).

The five rats injected with retinol all showed stage synchronization. The results on these five rats have been described in another paper (van Beek \& Meistrich, 1990). The probability of choosing at random four rats that would not become synchronized out of a group of nine rats of which the remaining five rats did become synchronized is 0.008 , or less than $1 \%$. We therefore conclude that the RD-RAM testes studied are representative of testes that will become stage synchronized upon injection of retinol.

\section{Control animals}

Rats were obtained from Harlan Sprague-Dawley (Indianapolis, IN). Two control rats were killed at 75, 111 or 203 days of age, weighing an average of 298,382 and $450 \mathrm{~g}$, respectively, in the three age groups. Rats of different ages were used spanning the age-range of both the depleted and the retinol-refed animals. The control material was used for calculation of the degree of synchrony and in some cases for cell counts. Additional quantitative data in cell counts were obtained from the extensive and detailed quantitative studies of Clermont \& Leblond (1953) and Huckins (1971).

\section{Histology - testicular cross-sections}

One testis of each rat was fixed in Bouin's solution. One part was mounted in paraffin, another part in plastic. Sections of $4 \mu \mathrm{m}$ of paraffin-embedded material and of $3 \mu \mathrm{m}$ of plastic-embedded material were stained by the periodic acid-Schiff reaction and counterstained with haematoxylin.

In sections of control testes, the stage of the cycle identified by the youngest generation of spermatids (Leblond \& Clermont, 1952b) aids in the identification of the various generations of differentiating spermatogonia. In the RDRAM testis, however, spermatids are absent, making an identification of the differentiating spermatogonia in the cross-sections more difficult. Spermatogonia were therefore classified as either A or Intermediate-type, or B-type. Early preleptotene spermatocytes are morphologically similar to B spermatogonia, but can be distinguished by their smaller size and, in general, they are more numerous in a given tubule region. 


\section{Histology - tubular whole mounts}

From the second testis of each animal, tubular whole mounts were prepared according to the method of Clermont \& Bustos-Orbregon (1968) and stained with haematoxylin. Although in tubular whole mounts it is not possible to stage each area accurately, the wave of the seminiferous epithelium, in which subsequent stages occupy adjacent areas of the seminiferous epithelium, makes identification of the differentiating spermatogonia possible. The spermatogenic wave along with the description of the cell morphology (Clermont \& Leblond, 1953) allowed identification of spermatogonia into A-, Intermediate- or B-type spermatogonia. Spermatogonia separated by an area of mitotic figures from B spermatogonia were considered to be Intermediate spermatogonia. Type A spermatogonia isolated in the seminiferous epithelium were considered to be undifferentiated $\mathbf{A}_{\text {single }}$ spermatogonia, as described by Huckins (1971). No further classification of type A spermatogonia was attempted.

In areas $5 \mathrm{~mm}$ apart in whole-mounted tubules, the number of cells per field was counted. In between each pair of these areas, nine fields were studied for mitoses. If there was more than one mitosis, it was ascertained whether they represented a clone of $A_{\text {paired }}$ or $A_{\text {aligned }}$ spermatogonia. In each RD-RAM animal, cells were counted in 34 areas in whole-mounted tubules; mitotic figures were studied in a total of 340 fields.

\section{Results}

The testes of the RD-RAM rats weighed $\sim 0.6 \mathrm{~g}$; control rats of similar age had testes of $\sim 1.6 \mathrm{~g}$. The body weight of the deficient rats at 98 days of age was $277 \pm 17 \mathrm{~g}$, less than that of controls of similar age. Figure 1 shows the growth of the four rats killed at 98 days of age that were on the RD-RAM diet.

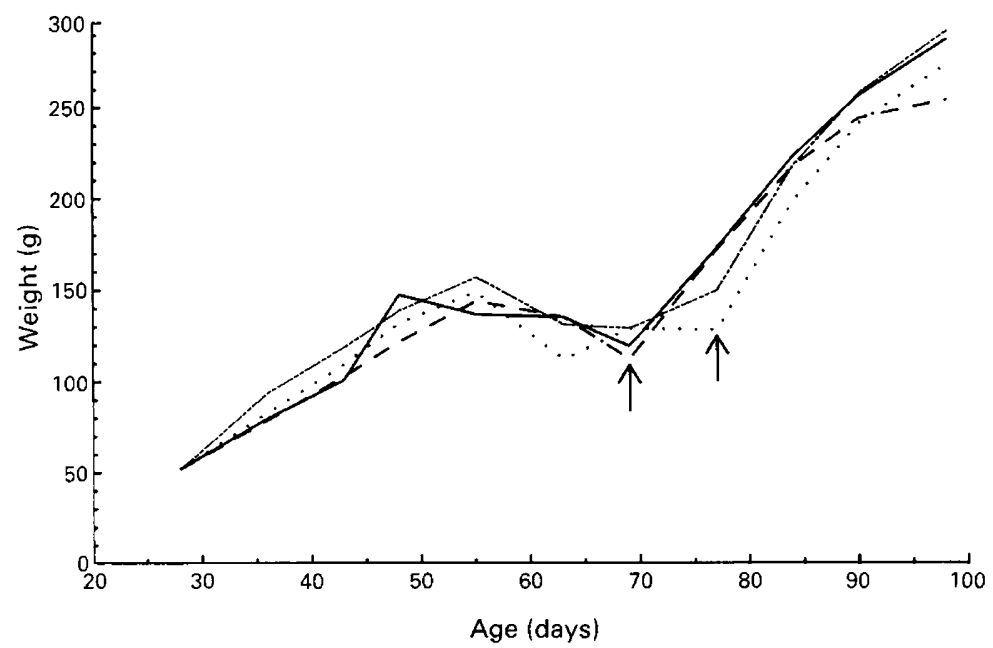

Fig. 1. Growth curves of four rats used in the histological analysis of the effects on the testes of retinol deficiency with retinoic acid maintenance. The rats were maintained on a totally retinoid-deficient diet from Day 20 and given retinoic acid maintenance at $69(---,-)$ or $77(\cdots,-\cdots-)$ days of age (arrows).

\section{Cross-sections}

General observations. A testicular cross-section from a control rat, showing portions of two tubules in stages VI and IX with normal B spermatogonia and leptotene spermatocytes, respectively, is shown in Fig. 2(a). All RD-RAM animals showed a severely regressed seminiferous epithelium (Fig. 2b and c). No normal cells at stages of development beyond preleptotene spermatocytes were found. However, aberrant cells with large pale nuclei containing widely dispersed strands of chromatin were seen in more luminal positions (Fig. 2c). Based on their position in the 
seminiferous epithelium, these cells would be classified as spermatocytes performing aberrant meiotic divisions. Type A spermatogonia were often seen in the same tubules.

Counts of tubule cross-sections with particular cell types. The percentage of tubule cross-sections totally depleted of spermatogenic cells and containing only Sertoli cells ranged between 3 and $34 \%$ (Table 1). In 14-30\% of the tubule cross-sections, the only spermatogenic cell types encountered were A or possibly Intermediate spermatogonia.

B spermatogonia were found, either alone or with A spermatogonia, in $7-19 \%$ of the tubule cross-sections, while preleptotene spermatocytes were encountered in $4-19 \%$ of the tubule crosssections. The sum of the tubule cross-sections that showed either B spermatogonia or preleptotene spermatocytes was $11-38 \%$. Although the percentage of tubule cross-sections showing B spermatogonia was in general approximately normal, in all rats the percentage of tubule cross-sections showing preleptotene spermatocytes was reduced to approximately half of control values or less. The numbers of $\mathbf{B}$ spermatogonia and preleptotene spermatocytes seen per tubule cross-section were variable and in general appeared lower than in control rats.

Aberrant cells were the only cell types seen, apart from Sertoli cells, in $13-25 \%$ of the tubule cross-sections; they were also seen together with A spermatogonia in $8-25 \%$ of the tubule crosssections, but not with B spermatogonia or preleptotene spermatocytes. Although quantitative differences between animals were observed, qualitatively the same features were observed in all animals studied.

\section{Tubular whole mounts}

General observations. Figure 2(d) shows a tubular whole mount of a control testis with dividing B spermatogonia and interphase A spermatogonia. The tubules of the retinol-deficient rats were rather thin because there were few spermatocytes and no spermatids (Fig. 2e and f). Areas with B spermatogonia were seen, as were areas containing preleptotene spermatocytes. These areas were often separated by a region containing mitotic figures (Fig. 2e). This was interpreted as the mitotic wave of B spermatogonia dividing into preleptotene spermatocytes, as is clearly the case in controls (Fig. 2d). Similarly, mitotic waves of Intermediate spermatogonia dividing into B spermatogonia were encountered, aiding in the identification of the Intermediate spermatogonia in the whole mounts. In regions of the seminiferous epithelium that did not contain Intermediate or B spermatogonia, groups of mitotic figures were regularly encountered; we believe that at least some of these correspond to groups of differentiating A spermatogonia dividing synchronously, as they do in control epithelium. Waves of divisions of these A spermatogonia, observed in control testes, were not so apparent in the deficient testes, probably because the density of the spermatogonia was severely reduced.

Along the length of the tubule the development of preleptotene spermatocytes into cells that resembled leptotene and zygotene spermatocytes could be followed. However, in control rats these cells were uniformly dark-staining, while in the deficient rats there seemed to be heterochromatic spots in the nuclei. Based on their position within the epithelium, it is likely that these abnormal leptotene and zygotene spermatocytes are the aberrant cells seen in tubule cross-sections (Fig. 2c). Degenerating cells of variable size were also seen in a more luminal position than spermatogonia, as shown in Fig. 2(f).

Cell counts. In tubular whole mounts of control animals, the density of Intermediate and B spermatogonia in those stages where they are present, is remarkably constant (Huckins, 1978). In the tubular whole mounts of RD-RAM testes, the density of the B spermatogonia, when they were present, was variable, and thus, the boundaries of areas where they should have been present in high numbers could not be established.

Only A spermatogonia were counted and their numbers expressed relative to those of the Sertoli cells (Table 2). No significant morphological differences between the spermatogonia in deficient and control rats were observed. Total numbers of A spermatogonia, given as a mean over the whole 

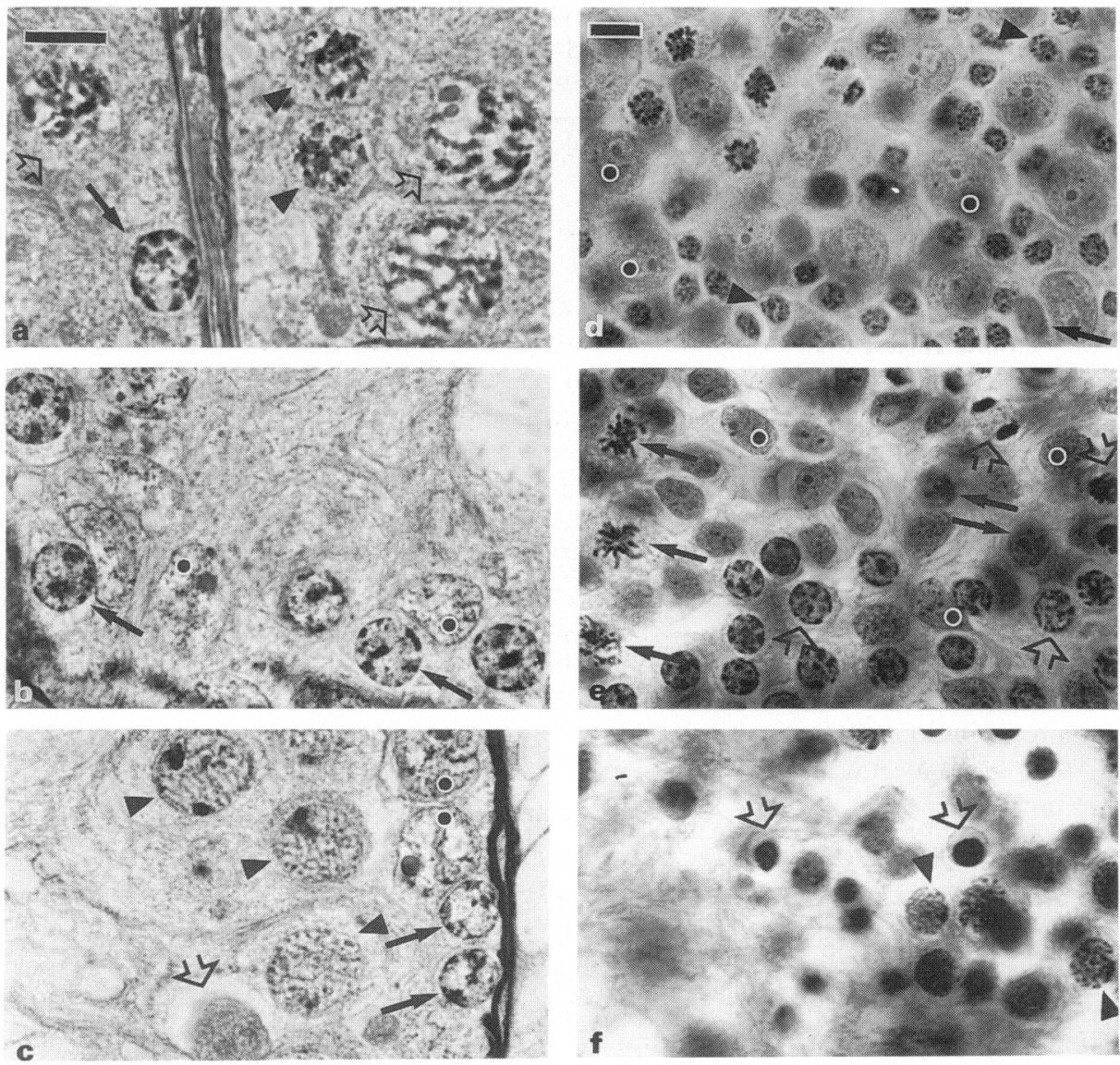

Fig. 2. Tubule cross-sections embedded in plastic (a-c) and whole mounts $(d-f)$, from testes of control ( $a$ and $d)$ and of retinol-deficient, retinoic acid-maintained $(b, c, e$ and $f)$ rats. For $(a-c)$ bar is $10 \mu \mathrm{m}$; for $(\mathrm{d}-\mathrm{f})$ bar is $10 \mu \mathrm{m}$.

(a) Two adjacent tubules, in stages VI and IX of the cycle of the seminiferous epithelium. Indicated are a B spermatogonium (arrow), young leptotene spermatocytes (arrowheads) and pachytene spermatocytes (open arrows). A Sertoli cell is located between the leptotene spermatocytes and the basement membrane, but the distinguishing features are out of focus. (b) A tubule of a deficient rat (rat no. 4), showing Sertoli cells (dots) and B spermatogonia (arrows). In the lower left corner a part of the basement membrane is visible.

(c) A tubule of a deficient rat (rat no. 4), showing Sertoli cells (dots), spermatogonia (arrows), aberrant spermatocytes (arrowheads), and a degenerating cell (open arrow). Based upon the morphology of neighbouring spermatogonia (not shown), the spermatogonia are probably A spermatogonia in telophase of mitosis or very early interphase.

(d) Control tubule in stage VI of the cycle of the seminiferous epithelium, showing the mitotic wave of the B spermatogonia. Sertoli cells (dots), A spermatogonia (arrow), and young preleptotene spermatocytes (arrowheads). The mitotic figures are all B spermatogonia.

(e) Tubule from a deficient rat (rat no. 1), focused on the basement membrane. Sertoli cells (dots), A spermatogonia, some that belong to a chain of eight in mitosis (arrows), and B spermatogonia (open arrows), some of which are in mitosis, are indicated.

(f) Tubule from a deficient rat (rat no. 1), focused on a level more towards the lumen than the nuclei of the Sertoli cells and spermatogonia. Aberrant prophasic cells (arrowheads), and degenerating cells (open arrows) are indicated. 
Table 1. Percentage of tubule cross-sections* in testes of retinoldeficient, retinoic acid-maintained rats showing different degrees of regression of the seminiferous epithelium

\begin{tabular}{|c|c|c|c|c|c|}
\hline \multirow[b]{2}{*}{ Spermatogenic cell stage } & \multicolumn{5}{|c|}{ Tubules $(\%)$ populated by indicated cell type } \\
\hline & Rat 1 & Rat 2 & Rat 3 & Rat 4 & Controls \\
\hline Sertoli cells only & 34 & 18 & 4 & 3 & 0 \\
\hline $\begin{array}{l}\text { A or Intermediate } \\
\text { spermatogonia }\end{array}$ & 30 & 14 & 29 & 22 & 0 \\
\hline B spermatogonia & 7 & 18 & 13 & 19 & 13 \\
\hline Preleptotene spermatocytes & 4 & 16 & 11 & 19 & 32 \\
\hline Aberrant cells only & 14 & 25 & 24 & 13 & 0 \\
\hline $\begin{array}{l}\text { Both A spermatogonia and } \\
\text { aberrant cells }\end{array}$ & 10 & 8 & 20 & 25 & 0 \\
\hline $\begin{array}{l}\text { Normal pachytene } \\
\text { spermatocytes }\end{array}$ & 0 & 0 & 0 & 0 & 100 \\
\hline
\end{tabular}

*501-614 tubule cross-sections/animal were studied in two sections.

epithelium, were low in all deficient rats, averaging $\sim 50 \%$ of control levels. The numbers of $\mathrm{A}_{\text {single }}$ spermatogonia were normal.

The mitotic indices, both for all $\mathrm{A}$ spermatogonia and for the $\mathrm{A}_{\text {single }}$ spermatogonia, were normal. The large mitotic figures encountered together with mitotic B spermatogonia (Fig. 2e) indicate mitoses of a chain of undifferentiated $\mathrm{A}_{\text {aligned }}$ spermatogonia.

\section{Epithelium after retinol replacement}

Three rats were killed 36 days after the first injection of retinol. In these rats, $89 \pm 6 \%$ of all tubule cross-sections were in stages VI, VII or VIII of the cycle of the seminiferous epithelium. In control rats, $37 \%$ of tubule cross-sections were found in these stages. Only $2 \cdot 6 \%$ of the tubule cross-sections in the synchronized rats were in stages X to II, while $43 \%$ of the tubule cross-sections in control rats are in these stages (van Beek \& Meistrich, 1990).

In the cross-sections of the synchronized testes, 22,41 and $56 \%$ of the tubules showed elongated spermatids in addition to round spermatids, while other tubules had round spermatids as the most advanced cell type. Elongated spermatids at steps 15 to 19 would have been between the stages of Intermediate spermatogonia and zygotene spermatocytes 36 days previously. In the RD-RAM rats, 11-38\% of the tubule cross-sections contained either B spermatogonia or preleptotene spermatocytes (Table 1). Thus, it seems that upon injection of retinol, B spermatogonia as well as preleptotene spermatocytes continued their development into elongated spermatids in almost all areas where they were present.

The testes of two rats, killed at 128 days after injection of retinol, still showed stage synchrony, although the degree of synchrony was lower than in those killed at 36 days after this injection (van Beek \& Meistrich, 1990). Additional rats from similarly treated groups, killed at intermediate intervals, confirm that stage synchrony can be routinely obtained with this protocol (van Beek \& Meistrich, 1991).

\section{Discussion}

Before the onset of weight loss due to retinol deficiency, rats develop a complete seminiferous epithelium (Mitranond et al., 1979). Four RD-RAM rats, randomly selected from a group of nine rats, all showed severely regressed seminiferous epithelium, although the degree of regression was variable. Nevertheless, in all four rats all spermatogonial cell types were observed, and were 
Table 2. Results of studies in whole mounted tubuli of retinol-deficient rats maintained on retinoic acid

\begin{tabular}{lccccc}
\hline & \multicolumn{5}{c}{ Rat } \\
\cline { 2 - 5 } Variable & 1 & 2 & 3 & 4 & Controls \\
\hline Number of Sertoli cells & 2048 & 1762 & 1931 & 1860 & - \\
$\quad$ counted & 89 & 123 & 177 & 128 & 250 \\
A $/ 1000$ Sertoli cells & 15 & 19 & 17 & 18 & $15-20^{\mathrm{a}}$ \\
$\mathrm{A}_{\text {single }} / 1000$ Sertoli cells & 1820 & 2170 & 3410 & 2390 & - \\
Number of A scanned & 47 & 25 & 79 & 37 & - \\
Number of mitoses & $2 \cdot 6$ & $1 \cdot 2$ & $2 \cdot 3$ & $1 \cdot 5$ & $1 \cdot 7-2 \cdot 0^{\mathrm{b}}$ \\
MI of A spermatogonia & 300 & 330 & 320 & 340 & - \\
Number of $A_{\text {single }}$ scanned & 7 & 3 & 5 & 3 & - \\
Number of isolated mitoses & $2 \cdot 3$ & $0 \cdot 9$ & $1 \cdot 6$ & $0 \cdot 9$ & $0 \cdot 14-2 \cdot 5^{\text {ac }}$ \\
MI of A $A_{\text {single }}$ spermatogonia & $2 \cdot 6$ & & & & \\
\hline
\end{tabular}

${ }^{a}$ Data derived from Huckins (1971).

${ }^{b}$ Calculated from data given by Clermont \& Leblond (1953); $1.7 \%$ refers to A spermatogonia, while $2.0 \%$ is the Mitotic Index (MI) of all spermatogonia during the cycle. Other values, ranging from $0.5 \%$ to $5.9 \%$ have been reported in the literature.

'In control animals, values vary during the cycle of the seminiferous epithelium, and are thus stage-dependent. Stages could not be identified in the deficient rats, hence the values given here are averages.

dividing. The remaining five rats all showed stage-synchronized seminiferous epithelium at 36 or 128 days after retinol injection (van Beek \& Meistrich, 1990).

\section{Spermatogonial cells in the RD-RAM testes}

Although the numbers of $A_{\text {single }}$ spermatogonia were normal in RD-RAM testes, the total number of A spermatogonia was only half that in control testes. In spite of severely reduced numbers of B spermatogonia, the percentage of tubule cross-sections showing at least some of these cells was close to normal.

The mitotic indices of $\mathrm{A}_{\text {single }}$ and of all $\mathrm{A}$ spermatogonia were in the normal range. Normal divisions of Intermediate and $B$ spermatogonia were observed in the RD-RAM animals. Divisions of $\mathrm{A}_{\text {aligned }}$ spermatogonia were also seen together with dividing $\mathrm{B}$ spermatogonia. In the control rats, B spermatogonia divided at stage VI of the cycle of the seminiferous epithelium, where $A$ spermatogonia did not divide. However, this continued proliferative activity of $\mathrm{A}_{\text {aligned }}$ spermatogonia up to stage VI does occur when the numbers of differentiating spermatogonia are reduced (de Rooij et al., 1985).

The present results are in agreement with previous studies on RD-RAM rats. Huang \& Hembree (1979) reported the presence of spermatogonia of all types, which incorporated $\left[{ }^{3} \mathrm{H}\right]$ thymidine and progressed into mitosis. Huang et al. (1990) found normal frequencies of tubule cross-sections showing different types of spermatogonia in RD-RAM rats. Unni et al. (1983) observed B-type spermatogonia in the RD-RAM epithelium and reported only a slightly lower mitotic index in RD-RAM rats than in controls. Thus in RD-RAM rats, there is no indication of a block in the proliferative activity of the A spermatogonia nor do these cells accumulate in a resting phase.

Retinoic acid apparently stimulates proliferation and differentiation of spermatogonia in the retinol-deficient rat. Recently, van Pelt \& de Rooij (1991) showed that retinoic acid, when given repeatedly and in high doses, could reinitiate spermatogenesis in a stage-synchronized fashion, as does retinol. The data presented in their study, obtained 36 and 49 days after initiation of retinoic acid treatment, suggest extensive cell loss between the generations of $B$ spermatogonia, pachytene 
spermatocytes, and round spermatids. In in vitro cultures of cryptorchid mouse testes, retinoic acid stimulated development of Intermediate and B-type spermatogonia, although retinol has a more pronounced effect than retinoic acid (Haneji et al., 1984). These studies also support the present observations that retinoic acid allows spermatogonial proliferation and development.

\section{Spermatocytes in the RD-RAM testes}

The only spermatocytes with normal morphology were the preleptotene cells. Although the percentage of tubule cross-sections showing B spermatogonia was normal, the percentage showing preleptotene spermatocytes was approximately half that of controls. Aberrant cells were also seen often in tubules containing A but not B spermatogonia. This distribution supports the identification of these cells as young but abnormal spermatocytes. Both in sections and in whole-mounted tubuli, degenerating cells were seen at a position in a luminal direction from the basement membrane. Thus, spermatocytes may begin to become aberrant approximately halfway through the preleptotene stage and ultimately degenerate.

Both the RD-RAM and the totally retinoid-deficient testis appear unable to support meiotic prophase. However, abnormal spermatocytes have not been described in totally retinoid-deficient rats.

\section{Implications for synchronization protocols}

The seminiferous epithelium of RD-RAM rats is regressed but asynchronous. However, 36 days after injection of retinol only a few stages (VI-VIII) have been observed (van Beek \& Meistrich, 1990). During these 36 days, some areas of the seminiferous epithelium must, therefore, have proceeded discontinuously, or at an altered rate, with the cycle of the seminiferous epithelium.

Results obtained with RD-RAM rats differ from recently published observations on the testes of retinol-deficient animals not maintained on retinoic acid (de Rooij et al., 1989; Griswold et al., 1989; Ismael et al., 1990; van Pelt \& de Rooij, 1990;). These investigators found undifferentiated A spermatogonia and preleptotene spermatocytes as essentially the only germ cells present and concluded that there is a block in the proliferation of the undifferentiated spermatogonia in the totally retinoid-deficient situation. It was proposed that injection of retinol would synchronize the entire epithelium by transforming all the blocked undifferentiated type $\mathrm{A}$ spermatogonia into $\mathrm{A}_{1}$ spermatogonia, a process which normally occurs in stages VII-VIII. Such a mechanism is insufficient to explain the synchrony observed in rats previously maintained on retinoic acid, where the continued presence of differentiating spermatogonia indicates that transformation of undifferentiated spermatogonia into differentiating spermatogonia was continually occurring.

The apparent restoration of spermatogenesis from stages VII-VIII (van Pelt \& de Rooij, 1990), even in the RD-RAM rats (van Beek \& Meistrich, 1991), suggests that an event peculiar to these stages is involved in the mechanism of synchronization. This could be the recruitment of undifferentiated spermatogonia to become $A_{1}$ spermatogonia. In these stages the preleptotene spermatocytes also undergo the final DNA-synthesis before entry into meiosis and pass through the Sertoli cell barrier. The presence and proliferation of $\mathrm{A}_{2}-\mathrm{B}$ spermatogonia, as observed in the RD-RAM testes, is not characteristic of stages VII-VIII. Initiation of stage synchrony must involve the establishment of the characteristics of the synchronized stages in most tubules, while eliminating possible signs of stages outside the stage-synchronized range. Huang et al. (1990) speculated that after retinol replacement, the proliferation of type $A_{2}-B$ spermatogonia in the RD-RAM testes might be suppressed, and Huang (1991) reported degeneration of $A_{4}$, Intermediate and $\mathrm{B}$ spermatogonia within $48 \mathrm{~h}$ of retinol replacement. Although retinol might act on different targets to produce proliferation of $\mathrm{A}_{\text {aligned }}$ spermatogonia, progression of meiosis, and degeneration of $\mathrm{A}_{2}-\mathrm{B}$ spermatogonia, a single target affecting these three endpoints could also be considered. 
The only cell that interacts with different germ cells is the Sertoli cell. Gap junctions are observed between germ cells and Sertoli cells (Russell, 1980), and recently, Mehta et al. (1989) found differential effects of retinoic acid and retinol on junctional intercellular communication. Sertoli cells also display a cyclic behaviour in their biochemical and structural properties related to the cycle of the seminiferous epithelium.

This leads to an alternative hypothesis to explain the synchronization in both the RD-RAM and the totally retinoid-deficient rats. It may be that retinol, but not retinoic acid, maintains or restores normal Sertoli cell function, and that in the absence of retinol the Sertoli cell cannot perform some critical function(s) that it normally would at stages VIl-VIIl. The injection of retinol in deficient rats restores the ability of the Sertoli cells to perform these functions, allowing not only the development of spermatocytes, but also stimulating the transformation of the undifferentiated type $A$ spermatogonia ( $A_{\text {paired }}$ and $A_{\text {aligned }}$ ) into $A_{1}$ spermatogonia. In the absence of the proper signals from the Sertoli cells, this transformation could still occur when retinoic acid is given, but at a lower efficiency. In the RD-RAM rats, just after retinol injection, the differentiated type A, Intermediate and B spermatogonia would be in stages out of phase with the Sertoli cell, and might degenerate because they are receiving inappropriate signals from the Sertoli cell. Their fate will have to be studied more carefully.

The above hypothesis is consistent with observations on both the totally retinoid-deficient and RD-RAM rats. However, further experiments are needed to test this concept.

We thank H. F. S. Huang for his advice on maintenance of retinol-deficient rats, C. R. Morales for his advice on the synchronizing protocol, S. L. Sivon for assistance with animal care and D. Goodman for secretarial assistance. Support for this research was provided by grants HD-16843 and CA-17364 from the National Institutes of Health. Histotechnical assistance was provided by the histopathological laboratories of the University of Texas M. D. Anderson Cancer Center, supported by core grant CA-16672.

\section{References}

Bartlett, J.M.S., Weinbauer, G.F. \& Nieschlag, E. (1989) Quantitative analysis of germ cell numbers and relation to intratesticular testosterone following vitamin A-induced synchronization of spermatogenesis in the rat. $J$. Endocr. 123, 403-412.

Bartlett, J.M.S., Weinbauer, G.F. \& Nieschlag, E. (1990) Stability of spermatogenic synchronization achieved by depletion and restoration of vitamin $A$ in rats. Biol. Reprod. 42, 603-612.

Clermont, Y. \& Bustos-Obregon, E. (1968) Reexamination of spermatogonial renewal in the rat by means of seminiferous tubules mounted 'in toto'. $\mathrm{Am}$. J. Anat. 122, 237-248.

Clermont, Y. \& Leblond, C. P. (1953) Renewal of spermatogonia in the rat. Am. J. Anat. 93, 475-501.

Coward, W.A., Howell, J. McC., Thompson, J.N. \& Pitt, G.A. (1969) The retinol requirement of rats for spermatogenesis and vision. Br.J. Nutr. 23, 619-626.

de Rooij, D.G., Lok, D. \& Weenk, D. (1985) Feedback regulation of the proliferation of the undifferentiated spermatogonia in the Chinese hamster by the differentiating spermatogonia. Cell Tissue Kinet. 18, 71-81.

de Rooij, D.G., van Dissel-Emiliani, F.M.F. \& van Pelt, A.M.M. (1989) Regulation of spermatogonial proliferation. Ann. N.Y. Acad. Sci. 564, 140-153.

Griswold, M.D., Bishop, P.D., Kim, K.-H., Ping, R., Siiteri, J.E. \& Morales, C. (1989) Function of vitamin
A in normal and synchronized seminiferous tubules. Ann. N.Y. Acad. Sci. 564, 154172.

Haneji, T., Maekawa, M. \& Nishimune, Y. (1984) Vitamin $\mathrm{A}$ and follicle-stimulating hormone synergistically induce differentiation of type A spermatogonia in adult mouse cryptorchid testes in vitro. Endocrinology 114, 801-805.

Howell, J.McC., Thompson, J.N. \& Pitt, G.A.J. (1963) Histology of the lesions produced in the reproductive tract of animals fed a diet deficient in vitamin $\mathrm{A}$ alcohol but containing vitamin $A$ acid. I. The male rat. J. Reprod. Fert. 5, 159-167.

Huang, H.F.S. (1991) Retinol-induced degeneration of spermatogonia in vitamin A deficient (VAD) rats. Proc. 11th North American Testis Workshop abstract 11.

Huang, H.F.S. \& Hembree, W.C. (1979) Spermatogenic response to vitamin $\mathrm{A}$ in vitamin $\mathrm{A}$ deficient rats. Biol. Reprod. 21, 891-904.

Huang, H.F.S., Marshall, G.R. \& Nieschlag, E. (1990) Enrichment of the stages of the seminiferous epithelium in vitamin A-replaced-vitamin A-deficient rats. J. Reprod. Fert. 88, 51-60.

Huckins, C. (1971) The spermatogonial stem cell population in adult rats. I. Their morphology, proliferation and maturation. Anat. Rec. 169, 533-558.

Huckins, C. (1978) The morphology and kinetics of spermatogonial degeneration in normal adult rats: 
an analysis using a simplified classification of the germinal epithelium. Anat. Rec. 190, 905926.

Ismael, N., Morales, C. \& Clermont, Y. (1990) Role of spermatogonia in the stage-synchronization of the seminiferous epithelium in vitamin-A-deficient rats. Am. J. Anat. 188, 57-63.

Leblond, C.P. \& Clermont, Y. (1952a) Spermiogenesis of rat, mouse, hamster and guinea pig as revealed by the 'periodic acid-fuchsin sulfurous acid' technique. $\mathrm{Am}$. J. Anat. 90, 167-215.

Leblond, C.P. \& Clermont, Y. (1952b) Definition of the stages of the cycle of the seminiferous epithelium in the rat. Ann. N.Y. Acad. Sci. 55, 548-573.

Mehta, P.P., Bertram, J.S. \& Loewenstein, W.R. (1989) The actions of retinoids on cellular growth correlate with their actions on gap junctional communication. J. Cell Biol. 108, 1053-1065.

Mitranond, V., Sobhon, P., Tosukhowong, P. \& Chindaduangat, W. (1979) Cytological changes in the testes of vitamin A deficient rats. I. Quantitation of germinal cells in the seminiferous tubules. Acta Anat. 103, $159-168$.

Morales, C. \& Griswold, M.D. (1987) Retinol-induced stage synchronization in seminiferous tubules of the rat. Endocrinology 121, 432-434.

Morales, C.R., Alcivar, A.A., Hecht, N.B. \& Griswold, M.D. (1989) Specific mRNAs in Sertoli and germinal cells of testes from stage synchronized rats. $\mathrm{Mol}$. Endocrinol. 3, 725-733.

Russell, L.D. (1980) Sertoli-germ cell interrelations: a review. Gamete Res. 3, 179-202.

Unni, E., Rao, M.R.S. \& Ganguly, J. (1983) Histological and ultrastructural studies on the effect of vitamin A depletion and subsequent repletion with vitamin A on germ cells and Sertoli cells in rat testis. Indian $J$. Exp. Biol. 21, 180-192.

van Beek, M.E.A.B. \& Meistrich, M.L. (1990) A method for quantifying synchrony in testes of rats, treated with vitamin A deprivation and readministration. Biol. Reprod. 42, 424-431.

van Beek, M.E.A.B. \& Meistrich, M.L. (1991) Stagesynchronized seminiferous epithelium in rats after manipulation of retinol levels. Biol. Reprod. 45, 235-244.

van Pelt, A.M.M. \& de Rooij, D.G. (1990) The origin of the synchronization of the seminiferous epithelium in vitamin A-deficient rats after vitamin A replacement. Biol. Reprod. 42, 677-682.

van Pelt, A.M.M. \& de Rooij, D.G. (1991) Retinoic acid is able to reinitiate spermatogenesis in vitamin Adeficient rats and high replicate doses support the full development of spermatogenic cells. Endocrinology 128, 697-704.

Received 7 December 1990 